\title{
Enhancing Visitor Experience or Hindering Docent Roles: Attentional Issues in Augmented Reality Supported Installations
}

\author{
Brandon Victor Syiem* Ryan M. Kelly ${ }^{\dagger} \quad$ Eduardo Velloso $\quad$ Jorge Goncalves ${ }^{\S} \quad$ Tilman Dingler ${ }^{\ddagger}$ \\ School of Computing and Information Systems \\ University of Melbourne"
}

\begin{abstract}
Studies using augmented reality (AR) technology have suggested that users focus excessively on the virtual content in the AR environment at the expense of the physical world around them. This has implications related to the design of installations that aim to incorporate the user's physical environment as part of the AR experience. To better understand how user attention is managed in an AR environment, we present an observational study of Rewild Our Planet, a multi-modal installation that combined video, audio, a human docent and mobile AR to promote awareness about environmental issues. We found that, while AR was successful in engaging visitors, it drew attention away from other modalities within the installation. This impacts the work of the human docent and affects how visitors absorb information presented in the installation. Based on these observations, we present guidelines to inform the design of future AR-supported installations with the aim of minimizing or taking advantage of the observed attentional issues.
\end{abstract}

Index Terms: Human-centered computing-Mixed / augmented reality; Human-centered computing-Empirical studies in HCI; Human-centered computing-Field studies.

\section{INTRODUCTION}

The appeal of augmented reality (AR) lies in its ability to present virtual content overlaid onto the physical world. This has resulted in the application of AR technology in settings such as games (Pokémon Go [7]), shopping applications (IKEA place [2]) and navigation (Google Maps AR). However, despite the juxtaposition of the physical and virtual, previous work has suggested that users focus primarily on the digital content, neglecting the physical world beyond it $[15,17,23]$. This leads to the question of how effective modern AR applications truly are in their use as a tool to 'augment' the physical world, as opposed to technology that replaces the physical world with a virtual one (as with Virtual Reality).

Although studies have noted the success of AR applications in enhancing user experience [18], engagement [50] and learning [23, $24,45]$, these studies have yet to question whether engagement with the AR technology comes at the cost of ignoring the user's physical environment. With the increasing availability of AR applications in the market, concerns related to user safety and situational awareness become critical. A simple scenario where allocation of user attention

\footnotetext{
*e-mail: bsyiem@student.unimelb.edu.au

†e-mail: ryan.kelly@unimelb.edu.au

†e-mail: eduardo.velloso@unimelb.edu.au

§e-mail: jorge.goncalves@unimelb.edu.au

Ile-mail: tilman.dingler@unimelb.edu.au

"This is the author's version of the work. It is posted here for your personal use. Not for redistribution. The definitive Version of Record was published in Proceedings of 2020 IEEE International Symposium on Mixed and Augmented Reality.
}

is especially important lies in the case of the Google Maps AR navigation tool: if the user is primarily focused on the virtual content, then there is a concern that the user may be oblivious to oncoming traffic.

Even in settings where safety is not the most critical factor, careful management of user attention remains important for the user experience. For example, in a study of a collaborative AR learning tool, Dunleavy et al. reported that students primarily focused on their handheld AR device, indicating a higher degree of engagement with presented content on the AR device [23]. However, the students were also observed to lose track of their physical environment, presenting a challenge in AR assisted learning should the experience be designed to incorporate learning resources beyond the application, such as the teacher. In such a case, the introduction of AR technology is just as likely to hinder as it is to augment the efforts of the teacher. However, there are studies in museum settings that demonstrate the ability for AR and mixed reality systems to work alongside human docents [61], suggesting that appropriate design of AR applications can support the efforts of the instructor. This leads us to question how the design of AR application can affect user attention and experience of the AR environment.

As a contribution towards understanding how design choices for AR applications can affect user attention and experience, we present findings from an in-the-wild study of Rewild Our Planet, a museum installation designed and created by a commercial software provider. The installation was multi-modal in its use of AR, video, audio and a human docent, to raise awareness about environmental issues through a social learning experience [6]. Given that the installation was designed to deliver content using multiple sources of information (both physical and virtual), this was an ideal scenario to explore how visitors managed their attention between the virtual and physical content.

During our observations, we discovered that the design of the installation, particularly the way in which AR was incorporated, affected both the visitor and the docent running the experience. First, we found that the docent was crucial in guiding visitor's attention within the installation, but had a challenging task in gaining and directing visitors' attention while the visitors were engaged with their AR device. Second, we observed that visitor attention was primarily focused on the AR content due to a phenomenon known as the attentional tunneling effect. Visitors were observed to neglect other channels such as the video, the docent and other visitors. This led to shallow engagement with the overall experience that left visitors with an impression of the technology rather than the core message of natural conservation the installation was trying to communicate. Finally, we found that the temporally and spatially separated nature of the content presented in the AR, video and audio led to the split attention effect, making it difficult for visitors to absorb the core message of the installation.

Our findings highlight the need to consider user attention when integrating AR into a multi-modal installations. We suggest that the design of multi-modal installations using AR should account for the 'attentional sink' that is caused by the AR content, so as to integrate content presented in all modalities. We present design guidelines 
to manage visitor attention and support the integration of AR into multi-modal installations.

\section{Related Work}

In this study, we focus on user attention in the context of a museum installation using AR technology. Given limited prior work done on how AR affects user attention, we discuss the broader topic of how digital displays affect user attention. We also discuss prior work focusing on how the introduction of technology affects the work of the docent.

\subsection{Digital Displays and their Effects on User Attention}

One of the first studies that reported a noticeable trend in user attention due to a display design was conducted by Fischer et al. [26]. The study compared pilot performance using a traditional HeadsDown Display (HDD) to a Heads-Up Display (HUD) to show flightrelevant information (altitude, flight path) [26] while a simulation of the outside world view was presented on a separate display in front of the pilot. While pilot performance was reported to be better using the HUD, pilots missed more unexpected events on the view of the outside world when using the HUD, as compared to the HDD. This was surprising as the HUD preserved the outside world in foveal view, while using the HDD required users to shift their viewpoint.

This phenomenon, where users excessively attend to one channel of information at the expense of missing events on other channels, was termed "Attentional Tunneling" and was formally defined by Wickens et al., [56] as: "The allocation of attention to a particular channel of information, diagnostic hypothesis, or task goal, for a duration that is longer than optimal, given the expected cost of neglecting events on other channels, failing to consider other hypotheses, or failing to perform other tasks"

Attentional Tunneling was initially attributed to HUD displays [26] but further research into the phenomenon has shown that it may have multiple causes. These include increased cognitive load [47,57], task priority and complexity [51], conversation [10], operator fatigue [41], and the priming effect [36]. It has also been speculated to be caused by novelty and infatuation with new technology [55].

A range of factors are thought to affect the degree to which Attentional Tunneling occurs. For instance, Wickens et al. [56] explored the attentional tunneling effect on two different displays presented on Head-Down Displays in an aviation setting. The first was a system simulating the aircraft's outside environment thereby previewing outside hazards (Synthetic Vision System or SV [46]), and the second was a 3D flight navigation system called Highway in the Sky (HITS) [1]. Attentional tunelling was observed to be more prominent on the HITS display when compared to the SV display. The authors attributed this to the design purpose of the displayswhile the SV was designed to inform users of the state of the outside world, the HITS display was designed to present information that implicitly or explicitly aimed to guide the user [56]. The authors also attributed the increased focus on the HITS display to the appeal of 3D displays.

An explanation as to why certain display designs caused attentional tunneling is related to how users perceptually group information [38]. HUD elements are presented on a separate depth layer to the background content (outside world view) and hence behave differently from the outside world view (for instance HUD components are stationary even when the outside view changes with respect to user motion). This prompts users to group the HUD elements and outside view as different planes of information and hence cannot efficiently divide their attention between the two planes [56]. A solution to this is to "Symbolically link" the HUD components to the view of the outside world, i.e. virtual components in the HUD were not stationary but changed relative to changes made by the user and the outside world [38]. This method has been shown to mitigate or completely eliminate the attentional tunneling effect $[37,38]$.

Modern AR technology has the capability of anchoring virtual objects to real objects, thereby fully registering their 3D position and orientation to the configuration of the physical environment. Though this should mitigate the attentional tunneling effect, studies of AR have suggested that users still exhibit attentional tunneling by focusing too much on the virtual objects and not the physical environment $[15,18,23]$. Dixon et al. explored the phenomenon in an experiment related to AR-assisted endoscopic navigation and concluded that AR does indeed cause attentional tunneling [20]. However, the AR used by Dixon et al. presented overlaid information, which led users to perceive the virtual information in a separate depth plane. This leads us to question if AR with anchored virtual content still causes this effect.

\subsection{AR Technology in Public Settings}

In this study, we examine the attentional tunneling effect and whether it is observed in users when introduced to modern AR technology in a museum setting. This setting provides an ideal scenario where visitors are not only exposed to the AR technology but their attention is also required by the physical exhibit/artefacts, other visitors and the docent. Another reason we consider a museum setting is due to the fact that AR technology also has an established history of being incorporated in museum settings. For example, Wojciechowski et al. [58] employed AR technology to enable visitors to view and interact with 3D models of fragile physical artefacts. A number of augmented guide systems have also been developed with the goal to cater museum tours to individual visitors [12,18, 19,42].

However, it is important to design these AR applications so that they do not entirely draw visitor attention away from from the physical artefact/exhibit. Chang et al. [18] recognized this issue and described the limitation of mobile AR-guide systems as: "[...] the inability to balance a visitor's attention distribution between the virtual space and the physical scenes, causing them to focus their attention on the "human-computer (guide system)" excessively, and to ignore the importance of the "human-field/situation (exhibition and local context)" in the real environment".

The docent is a part of the museum experience and one that competes for visitor attention when communicating information about the exhibition. A number of studies have indicated that docents play an integral role in shaping visitors' experience and that visitors view them as important sources of information [9, 29, 49, 59, 61]. Docents perform many tasks other than communicating the institutional message, such as guiding, informing and facilitating dialogue among participants [30]. The role of the docent, however, is often not formally prescribed [49], and a docent has to adapt to specific scenarios and enact multiple roles based on emergent circumstances. For instance, Yule et al. describe four different roles that a docent accommodates in their mixed reality game called Operation Citadel [61]. These roles are Guide, Interpreter, Social Facilitator and Performer. The docents would guide visitors through the mixed reality environment, interpret the instructions and the workings of their mixed reality game, facilitate discussion to solve tasks in the game, and perform as a character in the game world's narrative.

The nature of the exhibit can also determine the effectiveness of the message communicated by the docent. For instance, Mony et al. described a list of features including the nature of the exhibit, the duration of the interaction and the group composition of visitors, that can influence message communication in a zoo [43]. Introducing technology can also affect the nature of the exhibit, and this could, in turn, have a positive or negative impact on visitors' experience. Yule et al. noted that there is the potential to design for a symbiotic relationship between the human docent and accompanying technology, allowing the two to operate to enhance visitor experience and deliver key messages [61]. 


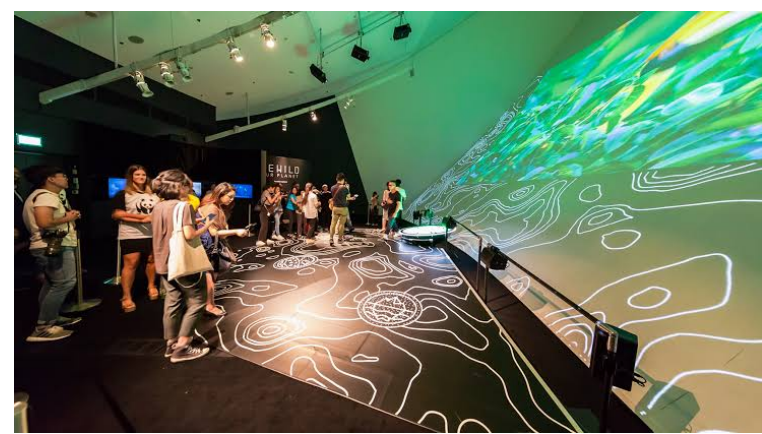

Figure 1: Layout of the Rewild Our Planet installation.

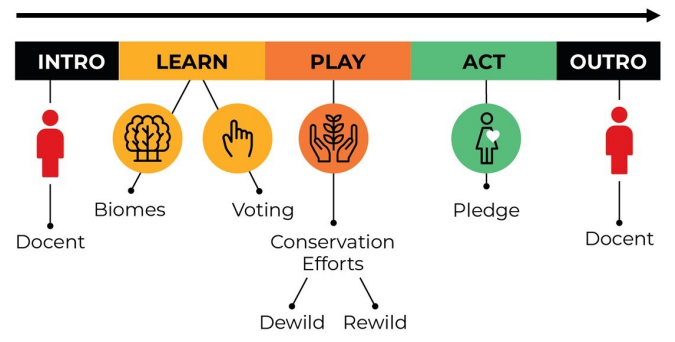

Figure 2: Diagram showing the journey of the user.

Prior work emphasises the interest and benefits of incorporating AR technology into museum exhibits and installations to enhance visitor experience, engagement and learning. There are, however, challenges to introducing novel technology into museums such as shallow engagement with the installation's core message [31]. Studies have also indicated that human docents can have an impact on visitor experience in mixed reality installations [61]. Our study aims to explore how the use of AR in an interactive installation influences the work of the docent and how it impacts visitors' experience.

\section{Rewild Our Planet}

Our findings are derived from an analysis of Rewild Our Planet (hereafter, 'Rewild'), an immersive installation based on the 2019 Netflix original documentary series Our Planet [3]. The Rewild installation was designed to tie in with the documentary series by using immersive content to educate visitors about humanity's role in the global decline of wildlife [4]. It achieved this by combining video, audio, and AR content to create a narrative in which groups of visitors used handheld mobile devices to bring life back to realistic 3D landscapes that appeared as elements within the story. The installation was designed and developed by a commercial software provider with a group of international partners. Our research team had no involvement in its design or development, meaning that we analysed the installation as outside observers.

The installation was deployed from April to June 2019 in three locations around the world. These were Dolby SoHo, which is a pop-up exhibition space in New York, USA; We The Curious, a science and arts centre in Bristol, UK; and the ArtScience Museum, a public museum at the Marina Bay Sands complex in Singapore.

\subsection{Installation Overview}

Rewild ran on a predefined schedule, with 20 minutes allotted to each run-through (three per hour). The installation supported a maximum of 10 simultaneous users, though it attracted varying numbers of people in each session, with groups varying from singletons to as

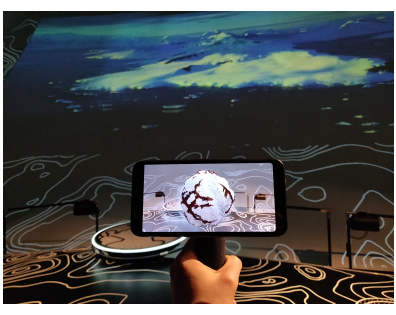

(a) Frozen introduction

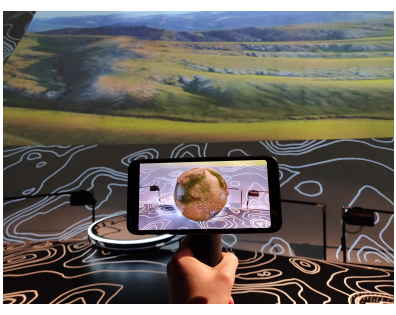

(b) Grasslands introduction.
Figure 3: Examples of AR content from the Learn section of Rewild Our Planet.

many as 15 people. Visitors shared a mobile handset in cases where not enough were available.

As shown in Figure 1, the installation was housed in a large room with low lighting. The room was equipped with speakers that played an ambient soundtrack between performances and which played sound during the experience. The installation was administered by a human docent, who welcomed guests to the experience and distributed the handsets that were used to view AR content.

The main physical components of the installation included a wallsized projection display, a circular pedestal called the "Crater", and a sloping wooden structure called the "Platform" (see Figure 1). The Platform served as the primary 'zone of engagement' [27] for the installation. Visitors were encouraged to cluster around and traverse the platform according to changes in the AR and video content. AR content was overlaid onto the Platform and above the Crater during the experience. This content was aligned to the exhibition space using proprietary software designed by the company behind Rewild.

The room also contained a dedicated server that handled all communication and synchronization between the mobile devices in the installation. This server was accessible to staff managing the installation in case of technical problems. Visitors were able to see the AR content using Google Pixel phones, which were handed to visitors by the docent before the experience. A maximum of 10 devices were available at each installation. Each phone was housed in a case that enabled visitors to hold the device by a handle. A master device was used to start the experience by the docent.

\subsection{Installation Content}

The Rewild installation used video and audio from the Our Planet documentary alongside a narrative voiceover from Sir David Attenborough. This was accompanied by custom AR content, created in response to the Our Planet Documentary. The overall narrative was driven by the video and the AR, with users prompted to move across the Platform by changes in the AR content. This meant that the experience was primarily cinematic, and visitors had minimal interaction with the AR content ${ }^{1}$.

The content within the installation was distributed over three main sections, that encouraged visitors to Learn, Play, and Act [5]. Figure 2 illustrates the visitors' journey through the installation, starting from an introduction delivered by the docent.

In the Learn section, the Crater was overlaid with an AR globe reflecting different biome textures (see Figure 3) with the aim of educating the visitors about a series of different natural biomes. Visitors could then vote to 'visit' one of these biomes by standing on a specific area of the platform, denoted by a graphic on the floor.

In the Play section, the platform was overlaid with textures of a natural environment - for example, a natural forest or arctic tundra-which visitors could explore by walking over the platform. This section also incorporated AR elements to demonstrate current

${ }^{1} \mathrm{~A}$ promotional video for the installation can be seen at https://www. youtube. com/watch?v=LGhQ7fYbYo4 


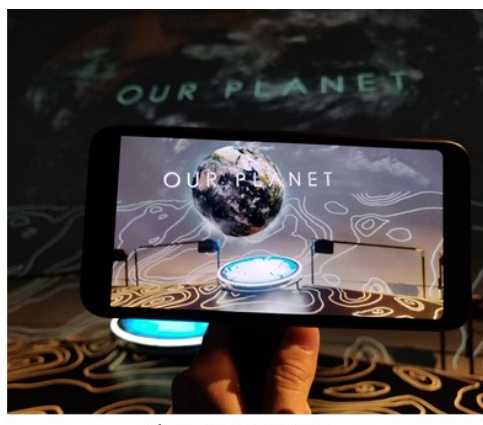

a) Opening Scene

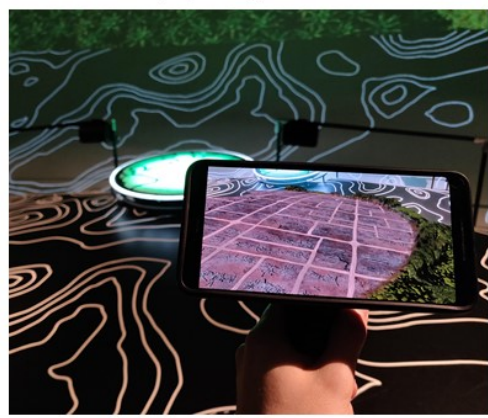

d) Dewild

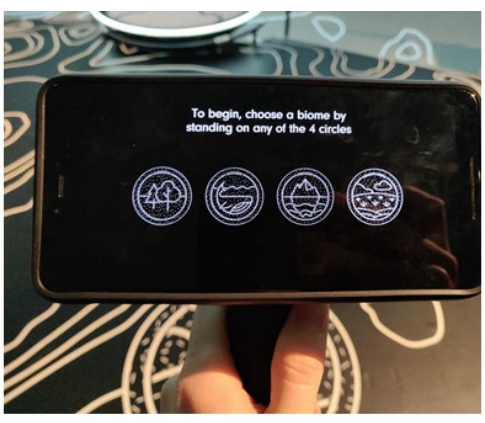

b) Selecting a biome

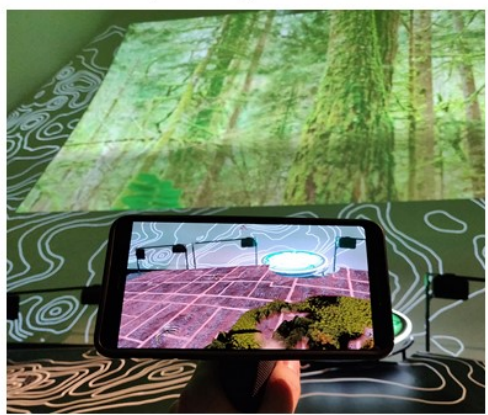

e) Rewild

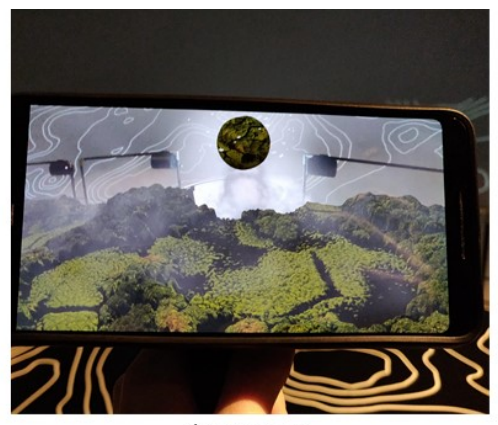

c) Exploring

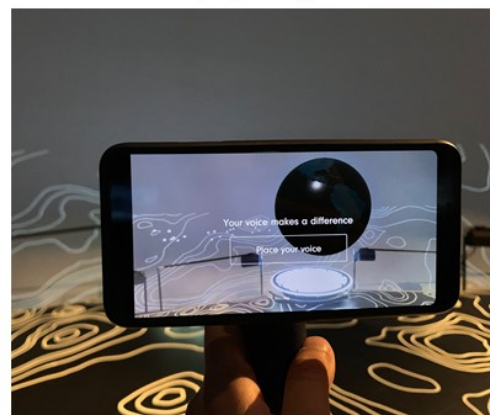

f) Pledge to save the environment

Figure 4: Figure showing AR elements at different sections of the experience. a) Introduction of Our Planet on the AR device within the Learn section. b) Biome selection instructions presented on the AR device within the Learn section. c) Exploring the forest biome during the Play section. d) Habitat loss during the 'Dewild' phase of the Play section. e) Restoring the virtual forest biome using the AR device during the Play section. f) Pledge to save the planet during the Act section.

environmental issues (called the "Dewild" phase) and the conservation efforts to ameliorate these issues (called the "Rewild" phase). In the Dewild phase, the virtual biome texture would change to reflect damage to that ecosystem, while in the Rewild phase, visitors could collectively "heal" the destroyed virtual biome by moving their AR device across the damaged texture, similar to using a paintbrush.

Finally, the Act section asked visitors to make an informal pledge to enact environmental change. Visitors saw a virtual globe hovering over the Crater (similar to the biome globes seen in Figure 3) and they were asked to trigger a metaphorical "voice" (represented by a shining sphere in the AR scene) by tapping a UI button on the AR device that showed their pledge as part of a global movement. This marked the end of the experience.

Figure 4 shows the changes in the AR content during the Learn, Play, and Act sections of the experience.

These three main sections were supported by a spoken introduction and outro, both of which were delivered by the docent. The docent's words helped to onboard users, gave more information on what the experience was about, and encouraged visitors to make change based on the experience. In addition, the docent helped visitors to calibrate the AR during the experience and swapped out mobile handsets in cases of technical difficulty.

\section{Methods}

We conducted an in-the-wild observational study [16] with the aim of understanding how the Rewild installation shaped visitors' attention and behaviour. We conducted our study over a two-week period in May 2019. This time period fell in the middle of Rewild's deployment. Two members of our research team spent a total of 44 hours in the field, comprising one day at the Bristol installation and five days at the Singapore installation. All of our materials and procedures received approval from our institution's ethical review panel before the study began. In addition, the research was conducted with the approval of museum staff overseeing the installation at each site.
We used a combination of first-hand experience, rapid ethnography [40] and informal interviews. These are established techniques for studying in-situ installations (e.g. [8,39,60]). First-hand experience involves using a technology of interest for oneself, and has been employed in prior studies of mobile AR [35] because it allows the research team to understand the experience from a user's perspective. In our case, we booked the first available slot at each site and we were taken through the installation alongside a group of visitors. This provided a grounded understanding of the user journey and allowed us to contextualise our findings in light of the installation's functionality. We also took photographs of the AR scenes in a later run-through to support our analyses.

We then engaged in ethnographic observations of visitors being taken through the experience by the docent. Our observations were conducted in a manner that avoided disrupting the flow of the experience for the visitors. We stood at the back of the installation room, approximately 10 metres from the platform. Once a session began, we approached the platform and made closer observations of visitors' behaviour. Our observations were focused on user behavior, social interactions between users and the docent, and the way in which visitors organised their attention during the experience. We observed 48 complete sessions and made field notes of salient observations from each session. Each session was run by a single docent and we observed a total of 5 individual docents running the sessions.

Finally, we conducted informal interviews with groups of visitors in order to collect further feedback about the experience. We did this approaching visitors as they were leaving the experience. 45 groups agreed to provide feedback about the experience. These comprised 6 individual users, 29 pairs of users and 10 groups of 3 or more users. The groups were distributed across sessions, and a maximum of two groups were interviewed independently by the researchers after each session. The interview questions were centered around understanding standout aspects of the user experience, what visitors liked and disliked, and what lessons they had taken away from the 
installation. As noted in previous work $[44,60]$, the nature of in-thewild research can make it challenging to conduct in-depth interviews. As such, the interviews lasted around 2-10 minutes and our written notes comprised salient points rather than verbatim transcripts.

\subsection{Analysis}

Our study produced 47 pages of field notes, 154 field photographs and 45 field interviews. Since our investigation relied primarily on ethnographic observations, we adopted an interpretivist stance in which we aimed to account for and describe phenomena that we observed in context [21]. We began by pooling our data and discussed salient observations as a team. Two authors then carried out a general inductive analysis [54] of the interview data, using independent parallel coding to categorise visitors' responses. This was followed by multiple sessions of whiteboarding and paper-based diagramming, both of which supported reflective discussion about how the design of Rewild impacted users' attention and engagement with the underlying message. The analytic process led us to a shared view of how the installation's design impacted the work of the docent, the behaviour of visitors, and how installations could make better use of AR to enhance the visitor experience.

\section{FINDINGS}

Our analysis identified three core issues related to the design of the installation. First, the installation itself relied heavily on the audio, video and AR content to guide visitors through the experience. This meant that the docent was not included by design to be a part of the installation. However, we observed that the docent adopted different roles to accommodate shortcomings of the installation in guiding visitors and encouraging social interactions. Second, the ability of the AR to 'grab' user attention led to the attentional tunneling effect whereby visitors primarily focused on the AR device and neglected other elements of the exhibit such as the video and the docent. Finally, the excessive focus on the AR device led to the split attention effect which caused issues related to how users absorbed and integrated information presented in the AR and the other modalities (video and the docent).

\subsection{The Docent and the Installation}

Rewild was designed as a standalone experience in which visitors were meant to be taken on a journey by the video, audio and AR, without the need for a human guide to become involved. For this purpose, the 2D display (prior to the start of the video) and the AR device provided instructional cues to the visitor on where to look and what to do during different phases of the experience. However, we observed that these instructions were insufficient and visitors required help from the docent. Visitors who did not understand how the installation proceeded would tend to disengage from the presented content.

We observed that the docent would take on different roles to ensure visitors were engaged with the content. We classified these roles according to the four categories described by Yule et al. [61]: Guide, Interpreter, Social Facilitator, Performer. In addition, we observed that the docents played an additional role of providing Technical support to manage the complexity introduced by the AR system. Each of these roles was crucial to manage different aspects of the user experience.

As a guide, the docent was tasked with organizing people in the space of the installation and directing them to points of interest. An example of this was seen when virtual fauna appeared on the terrain without any indication. We often saw the docent directing people to such events thereby ensuring visitors did not miss events that could potentially increase their enjoyment and engagement with the experience.

The docent also had to sometimes interpret the instructional cues provided by the AR device and video. For instance, during the 'Act' phase, visitors were instructed by the AR device to 'Place their voice' as a metaphorical pledge to save the environment. The device did not mention, however, that the pledge was actually a button that visitors had to tap for the experience to progress. We observed that the docent had to intervene in this stage and communicate to all visitors that they had to tap on the text on the screen.

The docent was seen facilitating social behaviour by acting as a Performer who was a part of the installation. For example, docents introduced a discussion section at the end of the experience with all visitors. This not only got visitors talking but also reinforced the message of the installation.

Lastly, the docent was also seen helping visitors with technical problems on their AR device. Examples include localization issues in which AR objects appeared in the wrong place, and inaccurate visitor positioning during the voting phase.

An observation we made when the docent was attempting to fulfill these roles was that they had difficulties attaining the visitors' attention. This was partly due to the loud and constant audio, rendering the docent's words nearly inaudible but mostly because the visitors were consistently focused on their AR device.

These integral roles that the docent undertook and the difficulty with which they accomplished these roles highlights the need to integrate the human operator into the design of complex installations using AR. The main crux in the Rewild installation was that every modality (Audio, Video and AR) was competing for user attention, making it difficult for the docent to carry out their roles.

\subsection{Visitors and the Installation}

Our second category of findings relates to how visitors managed their attention during the experience. Given the multi-modal nature of the exhibit and various information sources for the auditory and visual channels, it was immediately noticeable that visitors appeared to be overwhelmed by the amount of content. This was evident at the start of the experience during the 'Learn' phase when visitors were first presented with both the AR and video content. We observed visitors would take a step back from the Platform in an attempt to fit both the AR and video content within the AR device screen. However, this was not sustainable throughout the experience and visitors soon realized that they had to choose what to focus on.

\subsubsection{Attentional Tunneling}

Throughout our study, we noticed that visitors tended to focus on the mobile device presenting the AR, despite there being sections in the experience that presented no new virtual content. This led visitors to largely neglect the video and the docent, only occasionally looking up to the video display when there was a sudden change in contrast in the video or a shift in the audio. We aligned this behaviour with the Attentional Tunneling effect, which (as detailed in subsection 2.1), is a phenomenon where a user's attention is directed at a particular channel of information at the cost of neglecting other channels [56].

This viewpoint was reinforced in one of the first interviews we conducted where a participant mentioned that there was "too much focus on the mobile screen", making them "unsure where to look; screen (video) or mobile (AR)" (P4). Further interviews also reflected how effective the AR content was at 'grabbing' visitor attention. For example, 24 out of the 45 groups of visitors that we interviewed explicitly mentioned that the highlight of the experience was the AR component. Another 7 groups pointed out components of the AR application (virtual animals, interactive AR such as 'rewilding') as the highlight of their experience with Rewild Our Planet.

The implication of attentional tunneling for an installation like Rewild is that it may affect how visitors absorb information presented in other channels-in this case, the audio and video content. This was a concern, as most of the conservation-related messages were presented in the video and audio content. While the installation clearly succeeded in engaging visitors with the AR content at a 
superficial level, a problem lies in the fact that the AR content does not contain the core message of the installation. As we observed, the AR was meant to augment the content presented in the video and audio but overpowered their ability to attract the visitors' attention.

Causes of attentional tunneling: The attentional tunneling effect has been attributed to the design of digital displays and studies have reported that HUD displays [26,56] and 3D egocentric displays [25] cause the attentional tunneling effect. The presentation of the AR content on the AR device in Rewild bears similarities to a 3D egocentric view, however, the Rewild installation presents 3D content anchored to the outside/real view as opposed to the content being simply superimposed on the screen. This means that the user perceives the virtual content at a certain depth and the virtual content behaves accordingly as the users moves around in the space of the installation. This is an important distinction, as it has been shown that superimposed content causes the attentional tunneling effect while the presentation of virtual content in a similar depth plane to the outside/real view removes or mitigates the effect [38].

The anchoring of the virtual content in the installation, however, did not seem to remove the attentional tunneling effect completely and we still observed visitors solely attending to their AR device. Another cause for this effect relates to user expectation, which was geared towards the AR content, driven by the installation's marketing as a "Social Augmented Reality" experience. This leads to a priming effect in which prior knowledge of the system impacts user behaviour [32]. Kortschot et al. have demonstrated how priming users to expect events at certain locations on a screen could induce the attentional tunneling effect and reduce their reaction time to events in different locations [36]. This can be related to the Rewild installation, where visitors were "primed" to expect events on the AR device and thus miss events presented outside of the device. This was also reflected in responses to our question; "What brought you to this installation today?" which were largely directed towards the AR feature of the installation, e.g. "the Augmented Reality attracted us." (P15)

This expectation was also reinforced by the fact that visitors were handed out AR devices at the beginning of the experience, further reinforcing the priming effect and suggesting to them that the installation was to be experienced through this device.

Impact of attentional tunneling on content engagement: We observed that visitors used the AR device as a lens to experience the entire installation (including the video) rather than just a way to view the AR content. When a visitor's attention was drawn successfully away from the AR content by the docent or video, the visitor would view the docent or video through the AR device. A problem with this approach to viewing the installation, is the appeal of AR objects in the environment $[14,23,56]$ which immediately draws the user attention back to the AR content, almost rendering the other sources of information moot.

Impact of attentional tunneling on social interactions: The main goal of Rewild was to promote environmental awareness and spark discussion among the visitors. Although attentional tunneling did increase visitor engagement with the AR content, it appeared to adversely reduce social interactions. The multi-modal nature of installation also created a content dense experience, leaving little to no room for visitor-to-visitor or visitor-to-docent interactions. This prevented visitors from engaging with other visitors to reflect on and discuss the content being presented.

The experience attempted to promote social interaction and a sense of collaboration by using AR as a medium in the 'Rewild' section during the 'Play' phase. This section allows users to collaboratively rejuvenate a devastated biome in the AR environment, thereby fortuitously taking advantage of the attentional tunneling phenomenon. Collaboration, however, was not readily visible in our observations as the rewilding mechanic simply presented a shared view of the AR biome without any real need for the visitors to collaborate.

Even when no new content was presented in the AR device, visitors continued to focus their attention on the AR device as if expecting more content. We suspect the lack of guidance and feedback of where to focus their attention played a role in creating this situation. There was only a brief window after the rewild section that saw no new content on the AR device. This would be an ideal time for the docent to initiate a discussion amongst visitors about the message of the installation. However, the audio and video components continued to deliver content and the few visitors whose attention did move away from the AR device were now fixated on the video, preventing interactions with the docent or other visitors.

\subsubsection{Split Attention Effect on Installation Message}

The attentional tunneling effect meant that most visitors were more engaged with the AR content rather than the installation as a whole. This would not be a problem if the core message of the installation was presented in the AR device. However, we noticed that the AR device had been designed as a supporting technology to convey the messages presented in the audio and video components. We observed that the audio narrative was strongly linked to the video content and presented a cohesive coupling i.e., the video reflects what the audio narrative is trying to convey. However, the AR component does not match the video or audio content but rather attempts to support them by adding a simplified, "more immersive" and engaging version of the content.

This situation was where the attentional tunneling phenomenon caused an issue in the installation. Visitors were visually engaged with the AR content which was not well integrated to the audio narrative that they were hearing, this meant that visitors first needed to mentally integrate the messages from the visual and auditory channels to discern the message being presented. This is called the split attention effect, which occurs when two disparate sources of related information are spatially or temporally not well integrated [11]. For example, the 'Rewild' phase appeared in the AR when the audio/video content presented information on conservation efforts, however, it did not delve into the details of conservation as the audio/video did.

The split attention effect also occurred when the AR device presented instructional content while the video and audio sources were still delivering content related to the nature themes. This appeared to cause visitors to split their attention when trying to understand the AR instructions, absorbing the audio nature message and trying to integrate it with the contents of the AR device and video: “... (It was) difficult to look at the video and the AR at the same time. You want to see both but you end up missing it. If you are not using the device then you look more at the video. If (you are) using (the) phone, you look at the (AR device) screen all the time" (P36).

There were two main implications of the split attention effect. First, the effect makes it difficult for visitors to understand instructions presented on different sources of information. This makes visitors dependent on the docent to guide them through certain phases in the installation. For instance, during the voting phase, visitors were instructed by the AR device to cast a vote for a biome they wished to see. They could do this by standing on a biome marker, which were placed on a predetermined locations on the platform. As the visitors moved around from location-to-location on the platform, the 2D display also provided feedback on their current position and selected biomes. This spatially separated form of interaction often seemed to overwhelm and disengage visitors. We observed visitors moved minimally during the voting section as they were uncertain how this interaction worked in practice. It fell upon the docent to re-engage visitors with the installation, i.e., by trying to explain the voting mechanism and encouraging visitors to move around. In later sessions, we observed that the docent would explain the voting phase prior to the start of the experience in an 
attempt to prevent uncertainty during the voting phase. This seemed to work well and visitors who received this information were more engaged and mobile during the actual voting phase.

Second, through our interviews, we noticed that only 2 out of 45 visitor groups quoted a message from the audio or video content when asked about the message they took away from the experience. While this was not a definitive indicator that visitors failed to take away the environmental message, it does question if the message could have been delivered with more of an impact. We noticed that docents would try to reinforce the takeaways by reiterating some of the conservation messages at the end of the experience during the "Outro".

The interview data also reinforced our observations of the cohesiveness of the audio and video contents. This was especially clear in the case when participants had already seen the Our Planet documentary. One participant had this to say when we asked them about the message they took away from the experience: "I have seen Our Planet. I took away more from the documentary " (P10).

\section{Discussion}

Our objective in studying Rewild Our Planet was to understand how design choices for AR-supported installations can affect user attention and experience. The analysis of our observational and interview data led to three key findings.

First, we observed that the human docent played several emergent roles. These roles, although apparently not anticipated within the design of Rewild, were crucial for running the experience. The installation attempted to lead visitors through the experience via cues and instructions delivered by the AR device and video, yet we observed that these instructions proved insufficient and the docent had to step in to direct the experience. We also observed that the docent adopted different roles to guide visitors, interpret instructions, facilitate social behaviour, provide technical support, and offer contextual information in response to situations that arose within the experience. These observations are important because they reveal the variety of roles that docents can play in supporting complex AR installations, and suggest that future designs should aim for tighter integration of the human and technological components of the system.

Our second main observation was that visitors to Rewild were prone to the attentional tunneling effect and focused heavily on the AR content. This effect hindered the activities of the docent by adding an additional barrier in gaining visitors' attention, and caused users to become fixated on the AR at the expense of other channels. Attentional tunneling has been attributed to the design of AR displays $[18,20,23]$ and is known to lead users to neglect other channels of information [56]. In Rewild, attentional tunneling may have been increased by the 'priming' of visitors towards the AR elements, due to marketing efforts centered around AR. Users expected the experience to be mostly delivered through the AR device, and this appeared to direct their attention away from other modalities, further inhibiting social interaction between visitors and the docent.

Our third main observation was that the temporally and spatially separated information presented by the AR, video, and audio led to the split attention effect. We observed that split attention made it difficult for visitors to absorb the core message that the installation was trying to deliver. We also observed that the split attention effect stemmed from the excessive focus on the AR content, which was not well integrated with the audio and video.

These findings lead to questions on why attentional issues can emerge in AR-supported installations. One possible explanation is that the content-dense nature of Rewild, alongside the constant stream of information, may have forced visitors to selectively attend to certain elements to avoid information overload [33]. Phenomena such as attentional tunneling may therefore not be an artifact of AR itself, but of the extra effort made by users in trying to balance what they are attracted to, and what they think the expectations are from the exhibit. This issue could be avoided if the AR, audio and video were designed to complement each other (and the docent), without having to compete for user attention. An example of the successful integration of handheld AR in museum exhibitions can be seen in the CHESS project, where AR elements were embedded as story units in a larger story plot referenced around physical exhibits [34]. Another factor that could potentially cause attentional issues is the novelty of the AR technology itself $[31,55]$, and so designers should be aware of the 'wow factor' when considering how to integrate AR into visitor experiences.

Relatedly, problems with the integration of AR may arise if the design of an installation is not well aligned with the goals of the museum. However, disconnected design elements and unclear goals will always affect the outcome of exhibitions, not just those that integrate AR elements. We believe that misaligned goals were not the sole cause of the attentional issues we observed, given the extensive prior work suggesting that AR is prone to causing attentional problems $[18,20,23]$. Our findings provide insight into how attentional issues can emerge in the real world, and can help designers to mitigate similar outcomes in future deployments.

Future work could study a controlled variation of this installation to explore how the novelty of the AR technology could lead to the attentional tunneling effect. It could also aim to explore the installation from the designers' (and all stakeholders') perspective, as opposed to only the visitors'. This would give additional insights as to why certain design decisions were made and whether these decisions were aimed at the same goals.

\section{Design Guidelines}

Based on the findings of our analysis, we present five design guidelines to support the integration of AR into multi-modal installations.

\subsection{Incorporate the Human Component}

We found that the docent played a critical role in underpinning the experience by providing contextual information and guidance to the visitors. This was a challenging task due to the sheer amount of content in the installation that the docent had to work around to address the visitors. Other museum installations have had success in integrating the docent into the narrative of the installation by designing around them from the start $[22,61]$. This resulted in a seamless blend between the narrative delivered by the technology and the content delivered by the docent.

To address the challenges faced by the docent, we suggest foregrounding the role of the human operator in the design process and recognising their potential in driving experiences involving complex AR setups. This could be done by employing participatory design [53] to create the installation. Participatory design involves the co-design of technology in which the target users have a say in the technology design process. Previous work has included visitors as design partners to create museum exhibitions [53]. Future work should seek to involve docents in the design of the installation. Given that the docent uses the technology to deliver the core message of the installation, better integration between the technology and the docent can mitigate the challenges observed in our study. Taking one step further, the docent can also be included as a part of the narrative that the installation is trying to create, as done in Operation Citadel [61].

\subsection{Capitalise on Attention Tunneling}

Based on the observation of attentional tunneling, developers can anticipate that users will focus on the AR components and leverage this behaviour. Core content and installation messages should be delivered through the AR device and other sources of information 
should be used to support the content on the AR device. For example, the video could be used to complement the AR by providing contextual information while the AR presents the core message of the installation. An interesting example of such an implementation is the FoveAR, which uses Spatial AR to complement the limited field of view of Optical See-Through Head-Mounted AR device [13].

This also means that cueing methods should be employed in the AR application rather than outsourcing this task to a docent. This is because we observed that it was difficult for the docent to attract visitor attention once they were focused on the AR device.

\subsection{Mitigate Split Attention}

Through the implications of the split attention effect, we observed that visitors would either disengage because they did not understand the instructions or they did not grasp the environmental message of the installation. To ensure that these problems do not arise in future multi-modal installations, we suggest that disparate sources of related information should be spatially and temporally well integrated $[11,52]$. This is especially important when working with multi-display systems [48].

This means that the visual content should be temporally aligned with the audio content, i.e. the audio must reflect what the video is trying to convey at that point in time. It also means that related visual content should not be divided spatially between two different sources of information. For example, in the voting phase of Rewild Our Planet, instructions on how to select a biome was presented on the AR device (Figure 4b) while feedback on visitors' current locations were presented on the spatially separated wall display.

\subsection{Incorporate Purposeful Exploration}

We observed that visitors would be visibly pleased when finding points of interest in the AR environment. Digital wildlife was a recurring theme during our interview session and it was also observed to elicit the most dramatic response (visible and audible expressions) from visitors when the wildlife was discovered. This was not always the case however; in some sessions we observed users completely missing out on these points of interest due to a lack of attention guidance.

This brings us to the fourth design consideration: incorporating purposeful exploration. Discoverable elements were observed to add an incentive for users to explore the environment, which, in turn, increased user engagement. Though key points of interest requires guidance and are essential for the progression of the experience, discoverable elements are non-essential pieces that aim to increase immersion and user engagement. An example of adding discoverable elements can be seen in Gaitatzes et al's VR system, which enabled participants to explore different aspects of a rendered past civilization (such as people, their way of life, customs, etc.) as it progressed through time [28].

\subsection{Implement Collaborative Mechanics}

The Rewild Our Planet installation was envisioned as a "social augmented reality" experience that encouraged visitors to collaboratively work together, thereby sparking discourse on positive social change [5]. To enhance visitor participation and engagement, we suggest the inclusion of collaborative game mechanics into the installation that can be categorized into two different types: docentcentered collaboration and task-centered collaboration.

In docent-centered collaboration, the docent creates, controls, and facilitates a task that requires multiple visitors. For instance, giving the docent the ability to mould the AR environment or to create digital artefacts as points of discussion would simulate a "classroom" effect. This enables the docent to take on the role of a "teacher" while the visitors take on the role of "student" in discussing said artefact. In this type of collaboration, the docent acts as a prompt for visitor participation.
In task-centered collaboration, designers can encourage multiple users to form groups and work together to achieve a goal. This would be centered around a given goal with multiple subtasks. For example, in the "rewild" section, one user may be given the task to "heal" the digital biome, while other users are required to use a different game mechanic to stop the destructive elements from reclaiming the "healed" sections. In this type of collaboration, other visitors would act as prompts for participation if they need help to complete the task.

\section{Conclusion}

This study shows how the introduction of AR technology into a multi-modal installation impacts the visitors and the docent running the experience. It highlights the need to consider user attention when content is distributed in multiple modalities and also how AR technology can affect the work of a human docent or instructor in such settings. Based on our findings, we present a set of guidelines which aim to minimize the adverse effects of attentional issues related to mobile AR use and support the work of the docent in a multi-modal installation. We argue that understanding user attention when designing AR-supported installations is crucial to improve user experience and engagement with the content, thereby supporting message delivery and providing a satisfying user experience.

\section{ACKNOWLEDGMENTS}

The authors wish to thank everyone at PHORIA and partners for instigating the Rewild Our Planet installation. The authors also wish to thank the staff at We the Curious and the ArtScience Museum for their assistance and cooperation during the study.

\section{REFERENCES}

[1] Highway in the sky. https://www.hq.nasa.gov/office/aero/ docs/chicago/hits.htm.

[2] Ikea place. https://www.ikea.com/au/en/apps/IKEAPlace. html, 2014.

[3] Our planet, netflix. https://www.netflix.com/au/title/ 80049832, 2019.

[4] Rewild our planet. https://www.marinabaysands.com/museum/ rewild-our-planet.html, 2019.

[5] Rewild our planet. https://drivenxdesign.com/now/project. asp?ID=18121, 2019.

[6] Rewild our planet, phoria. https://www.phoria.com.au/ projects/wwf-rewild, 2019.

[7] Niantic, Inc. Pokémon go. https://www. pokemongo.com, 2016.

[8] I. Akpan, P. Marshall, J. Bird, and D. Harrison. Exploring the effects of space and place on engagement with an interactive installation. In Proceedings of the SIGCHI Conference on Human Factors in Computing Systems, CHI '13, pp. 2213-2222. ACM, New York, NY, USA, 2013. doi: $10.1145 / 2470654.2481306$

[9] J. R. Anderson, L. M. Reder, and H. A. Simon. Situated learning and education. Educational researcher, 25(4):5-11, 1996. doi: 10. 2307/1176775

[10] P. Atchley and J. Dressel. Conversation limits the functional field of view. Human factors, 46(4):664-673, 2004. doi: 10.1518/hfes.46.4. 664.56808

[11] P. Ayres and J. Sweller. The split-attention principle in multimedia learning. The Cambridge handbook of multimedia learning, 2:135-146, 2005.

[12] B. B. Bederson. Audio augmented reality: A prototype automated tour guide. In Conference Companion on Human Factors in Computing Systems, CHI '95, pp. 210-211. ACM, New York, NY, USA, 1995. doi: $10.1145 / 223355.223526$

[13] H. Benko, E. Ofek, F. Zheng, and A. D. Wilson. Fovear: Combining an optically see-through near-eye display with projector-based spatial augmented reality. In Proceedings of the 28th Annual ACM Symposium on User Interface Software \& Technology, pp. 129-135, 2015.

[14] M. Billinghurst, D. Belcher, A. Gupta, and K. Kiyokawa. Communication behaviors in colocated collaborative ar interfaces. International 
Journal of Human-Computer Interaction, 16(3):395-423, 2003. doi: 10.1207/S15327590IJHC1603_2

[15] M. Billinghurst and H. Kato. Collaborative augmented reality. Communications of the ACM, 45(7):64-70, 2002

[16] B. Brown, S. Reeves, and S. Sherwood. Into the wild: Challenges and opportunities for field trial methods. In Proceedings of the SIGCHI Conference on Human Factors in Computing Systems, CHI '11, pp. 1657-1666. ACM, New York, NY, USA, 2011. doi: 10.1145/1978942. 1979185

[17] G. Chang, P. Morreale, and P. Medicherla. Applications of augmented reality systems in education. In Society for Information Technology \& Teacher Education International Conference, pp. 1380-1385. Association for the Advancement of Computing in Education (AACE), 2010.

[18] K.-E. Chang, C.-T. Chang, H.-T. Hou, Y.-T. Sung, H.-L. Chao, and C.M. Lee. Development and behavioral pattern analysis of a mobile guide system with augmented reality for painting appreciation instruction in an art museum. Computers \& Education, 71:185-197, 2014. doi: 10. 1016/j.compedu.2013.09.022

[19] A. Damala. Interaction Design and Evaluation of Mobile Guides for the Museum Visit: A Case Study in Multimedia and Mobile Augmented Reality. PhD thesis, 2009.

[20] B. J. Dixon, M. J. Daly, H. Chan, A. D. Vescan, I. J. Witterick, and J. C. Irish. Surgeons blinded by enhanced navigation: the effect of augmented reality on attention. Surgical endoscopy, 27(2):454-461, 2013.

[21] P. Dourish. Reading and interpreting ethnography. In Ways of Knowing in HCI, pp. 1-23. Springer, 2014. doi: 10.1007/978-1-4939-0378-8_1

[22] S. Dow and B. MacIntyre. Experiences employing novice wizard operators in a gallery setting. In International Conference on Entertainment Computing, pp. 190-196. Springer, 2008.

[23] M. Dunleavy, C. Dede, and R. Mitchell. Affordances and limitations of immersive participatory augmented reality simulations for teaching and learning. Journal of science Education and Technology, 18(1):7-22, 2009. doi: 10.1007/s10956-008-9119-1

[24] L. Escobedo, M. Tentori, E. Quintana, J. Favela, and D. Garcia-Rosas. Using augmented reality to help children with autism stay focused. IEEE Pervasive Computing, 13(1):38-46, 2014. doi: 10.1109/MPRV. 2014.19

[25] S. Fadden, P. M. Ververs, and C. D. Wickens. Pathway huds: Are they viable? Human Factors, 43(2):173-193, 2001. PMID: 11592660. doi $10.1518 / 001872001775900841$

[26] E. Fischer and R. F. Haines. Cognitive issues in head-up displays. 1980.

[27] P. T. Fischer and E. Hornecker. Urban hci: Spatial aspects in the design of shared encounters for media facades. In Proceedings of the SIGCHI Conference on Human Factors in Computing Systems, CHI '12, pp. 307-316. ACM, New York, NY, USA, 2012. doi: 10.1145/2207676. 2207719

[28] A. Gaitatzes, D. Christopoulos, and M. Roussou. Reviving the past: cultural heritage meets virtual reality. In Proceedings of the 2001 conference on Virtual reality, archeology, and cultural heritage, pp. 103-110, 2001.

[29] T. Gärling, A. Biel, and M. Gustafsson. The new environmental psychology: The human interdependence paradigm. Handbook of environmental psychology, pp. 85-94, 2002.

[30] R. S. Grenier. Taking the lead: a qualitative study of expert docent characteristics. Museum Management and Curatorship, 26(4):339-353, 2011. doi: 10.1080/09647775.2011.603929

[31] E. Hornecker. "i don't understand it either, but it is cool"-visitor interactions with a multi-touch table in a museum. In 2008 3rd IEEE international workshop on horizontal interactive human computer systems, pp. 113-120. IEEE, 2008. doi: 10.1109/TABLETOP.2008. 4660193

[32] K. Jokinen and T. Hurtig. User expectations and real experience on a multimodal interactive system. In Ninth International Conference on Spoken Language Processing, 2006.

[33] S. L. Jones and R. Kelly. Dealing with information overload in multifaceted personal informatics systems. Human-Computer Interaction, 33(1):1-48, 2018.
[34] J. Keil, L. Pujol, M. Roussou, T. Engelke, M. Schmitt, U. Bockholt, and S. Eleftheratou. A digital look at physical museum exhibits: Designing personalized stories with handheld augmented reality in museums. In 2013 Digital Heritage International Congress (DigitalHeritage), vol. 2 , pp. 685-688. IEEE, 2013.

[35] R. M. Kelly, H. S. Ferdous, N. Wouters, and F. Vetere. Can mobile augmented reality stimulate a honeypot effect? observations from santa's lil helper. In Proceedings of the 2019 CHI Conference on Human Factors in Computing Systems, CHI '19, pp. 285:1-285:13. ACM, New York, NY, USA, 2019. doi: 10.1145/3290605.3300515

[36] S. W. Kortschot and G. A. Jamieson. Classification of attentional tunneling through behavioral indices. Human factors, p. 0018720819857266 , 2019. doi: $10.1177 / 0018720819857266$

[37] J. Lasswell and C. Wickens. The effects of display location and dimensionality on taxi-way navigation (tech. rep. no. arl-95-5/nasa-95-2). Savoy: University of Illinois, Aviation Research Laboratory, 1995.

[38] J. L. Levy, D. C. Foyle, and R. S. McCann. Performance benefits with scene-linked hud symbology: an attentional phenomenon? In Proceedings of the Human Factors and Ergonomics Society Annual Meeting, vol. 42, pp. 11-15. SAGE Publications Sage CA: Los Angeles, CA, 1998. doi: $10.1177 / 154193129804200104$

[39] P. Marshall, Y. Rogers, and N. Pantidi. Using f-formations to analyse spatial patterns of interaction in physical environments. In Proceedings of the ACM 2011 Conference on Computer Supported Cooperative Work, CSCW '11, pp. 445-454. ACM, New York, NY, USA, 2011. doi $10.1145 / 1958824.1958893$

[40] D. R. Millen. Rapid ethnography: Time deepening strategies for hci field research. In Proceedings of the 3rd Conference on Designing Interactive Systems: Processes, Practices, Methods, and Techniques, DIS '00, pp. 280-286. ACM, New York, NY, USA, 2000. doi: 10. $1145 / 347642.347763$

[41] K. C. Mills, S. E. Spruill, R. W. Kanne, K. M. Parkman, and Y. Zhang. The influence of stimulants, sedatives, and fatigue on tunnel vision: risk factors for driving and piloting. Human factors, 43(2):310-327, 2001. doi: 10.1518/001872001775900878

[42] T. Miyashita, P. Meier, T. Tachikawa, S. Orlic, T. Eble, V. Scholz, A. Gapel, O. Gerl, S. Arnaudov, and S. Lieberknecht. An augmented reality museum guide. In Proceedings of the 7th IEEE/ACM International Symposium on Mixed and Augmented Reality, pp. 103-106. IEEE Computer Society, 2008. doi: 10.1109/ISMAR.2008.4637334

[43] P. R. Mony and J. E. Heimlich. Talking to visitors about conservation: Exploring message communication through docent-visitor interactions at zoos. Visitor Studies, 11(2):151-162, 2008. doi: 10. 1080/10645570802355513

[44] K. O'Hara, M. Glancy, and S. Robertshaw. Understanding collective play in an urban screen game. In Proceedings of the 2008 ACM Conference on Computer Supported Cooperative Work, CSCW '08, pp. 67-76. ACM, New York, NY, USA, 2008. doi: 10.1145/1460563. 1460576

[45] L. Pemberton and M. Winter. Collaborative augmented reality in schools. In Proceedings of the 9th international conference on Computer supported collaborative learning-Volume 2, pp. 109-111. International Society of the Learning Sciences, 2009.

[46] L. Prinzel and L. Kramer. Synthetic vision systems. 2009.

[47] E. M. Rantanen and J. H. Goldberg. The effect of mental workload on the visual field size and shape. Ergonomics, 42(6):816-834, 1999. doi: $10.1080 / 001401399185315$

[48] U. Rashid, M. A. Nacenta, and A. Quigley. Factors influencing visual attention switch in multi-display user interfaces: A survey. In Proceedings of the 2012 international symposium on pervasive displays, pp. $1-6,2012$.

[49] W. B. Rayward and M. B. Twidale. From docent to cyberdocent: Education and guidance in the virtual museum. Archives and Museum Informatics, 13(1):23-53, 1999. doi: 10.1023/A:1009089906902

[50] S. Reeves, M. Fraser, H. Schnadelbach, and S. Benford. Engaging augmented reality in public places. In Adjunct Proceedings of the SIGCHI Conference on Human Factors in Computing Systems, CHI '05. ACM, New York, NY, USA, 2005.

[51] J. Roge, L. Kielbasa, and A. Muzet. Deformation of the useful visual field with state of vigilance, task priority, and central task complexity. 
Perceptual and motor skills, 95(1):118-130, 2002. doi: 10.2466/pms. 2002.95.1.118

[52] P. Sommerauer and O. Müller. Augmented reality in informal learning environments: A field experiment in a mathematics exhibition. Com puters \& Education, 79:59-68, 2014. doi: 10.1016/j.compedu.2014. 07.013

[53] G. Taxén. Introducing participatory design in museums. In Proceedings of the Eighth Conference on Participatory Design: Artful Integration: Interweaving Media, Materials and Practices - Volume 1, PDC 04, pp. 204-213. ACM, New York, NY, USA, 2004. doi: 10.1145/1011870. 1011894

[54] D. R. Thomas. A general inductive approach for analyzing qualitative evaluation data. American Journal of Evaluation, 27(2):237-246, June 2006. doi: $10.1177 / 1098214005283748$

[55] C. D. Wickens. Attentional tunneling and task management. In 2005 International Symposium on Aviation Psychology, p. 812, 2005.

[56] C. D. Wickens and A. L. Alexander. Attentional tunneling and task management in synthetic vision displays. The International Journal of Aviation Psychology, 19(2):182-199, 2009. doi: 10.1080/ 10508410902766549
[57] L. Williams. Visual field narrowing induced by workload. J Gen Psychol, 122:225-235, 1995.

[58] R. Wojciechowski, K. Walczak, M. White, and W. Cellary. Building virtual and augmented reality museum exhibitions. In Proceedings of the ninth international conference on 3D Web technology, pp. 135-144. ACM, 2004. doi: 10.1145/985040.985060

[59] R. L. Wolf and B. L. Tymitz. ” do giraffes ever sit?": A study of visitor perceptions at the national zoological park, smithsonian institution. 1979.

[60] N. Wouters, J. Downs, M. Harrop, T. Cox, E. Oliveira, S. Webber, F. Vetere, and A. Vande Moere. Uncovering the honeypot effect: How audiences engage with public interactive systems. In Proceedings of the 2016 ACM Conference on Designing Interactive Systems, DIS '16, pp. 5-16. ACM, New York, NY, USA, 2016. doi: 10.1145/2901790. 2901796

[61] D. Yule, B. MacKay, and D. Reilly. Operation citadel: Exploring the role of docents in mixed reality. In Proceedings of the 2015 Annual Symposium on Computer-Human Interaction in Play, CHI PLAY '15, pp. 285-294. ACM, New York, NY, USA, 2015. doi: 10.1145/2793107 .2793135 